\title{
Intraoperative neuromonitoring of hypogastric plexus branches during surgery for rectal cancer-preliminary report
}

\author{
Authors' Contribution \\ A-Study Design \\ B-Data Collection \\ C-Statistical Analysis \\ D-Data Interpretation \\ E-Manuscript Preparation \\ F-Literature Search \\ G-Funds Collection \\ Wałęga PiotraBEFC, Romaniszyn Michał ${ }^{\text {ACDE }}$, Wałęga Maciej ${ }^{\text {BCE }}$, Świrta Szymon ${ }^{\text {CDEF, }}$ \\ Nowak Wojciech ${ }^{A}$ \\ III Katedra i Klinika Chirurgii Ogólnej UJCM Oddział Kliniczny Chirurgii Ogólnej Szpitala Miejskiego Specjalistycznego \\ im G. Narutowicza
}

Article history: Received: 22.03.2016 Accepted:03.03.2017 Published:30.04.2017

ABSTRACT:

Aim: The aim of this study was to present our preliminary experience with intraoperative neuromonitoring during rectal resection.

Materials and methods: We qualified 4 patients ( 2 women, 2 men; age $42-53$ years) with rectal cancer for surgery with intraoperative neuromonitoring. In all patients, functional tests of the anorectal area were performed before surgery. Action potentials from the sphincter complex in response to nerve fiber stimulation were recorded with electrodes implanted before surgery. Moreover, we inserted a standard, 18FR Foley's urinary catheter to which a T-tube was connected to allow urine outflow and measurement of pressure changes in the bladder induced by detrusor contractions during stimulation.

Results: Setting up neuromonitoring prolonged surgery time by 30 to 40 minutes, or even by 60 to 80 minutes in the case of the first two patients. Neuromonitoring itself took additional 20 to 30 minutes during surgery. In all patients, we stimulated branches of the inferior hypogastric plexus in their anatomical position during dissection. In three patients, we evoked responses both from the bladder and the sphincter in all planes of stimulation. In one patient, there was no response from the left side of the bladder, and in the same patient, we observed symptoms of neurogenic bladder.

Conclusions: Based on the available literature and our own experience, we state that monitoring of bladder pressure and electromyographic signals from rectal sphincters enables visualization and preservation of autonomic nervous system structures, both sympathetic and parasympathetic. Intraoperative signals seem to be correlated with clinical presentation and functional examinations after surgery. In order to objectify our results, it is necessary to perform functional examinations before and after surgery in a larger group of patients.

KEYWORDS: $\quad$ rectal cancer, neuromonitoring, rectal resection, complications

\section{INTRODUCTION}

Functional complications of rectal surgery decrease quality of life due to an impaired control over basic physiological functions. Total mesorectal excision (TME), introduced in 1982 by Heald, is the mainstay of treatment for rectal cancer that enables complete tumor resection $(1,2)$. However, despite nerve sparing techniques that are applied during TME, in 50-70\% of patients, TME is associated with damage to nerves in the inferior and superior hypogastric plexuses and other nerves that control the bladder, genitals, rectum, and the rectal sphincter complex. Nerve damage is more likely when dissecting the anterior or lateral rectal wall, especially in patients with narrow pelvises, bleeding, or unfavorable tumor locations. As regards laparoscopic procedures, although magnification of the surgical fields makes it easier to identify nerve structures, the risk of functional complications is similar as in the case of standard surgery.

Anterior resection syndrome is a well-known complication in which rectal sphincter dysfunction is accompanied by disorders of micturition and sexual function (3).These impairments severely decrease quality of life in affected patients. High costs of managing such complications and the necessity to form stomata in some patients call for new approaches that could reduce the risk of anterior resection syndrome. One of such approaches involves identification of nerve structures during surgery. First reports on neuromonitoring reach as far as 1898, when Krause used electrostimulation of the facial nerve with facial muscle responses during resection of the vestibulocochlear nerve (4). Currently, neuromonitoring is successfully used in thyroid gland surgery, laryngology, vascular surgery, and

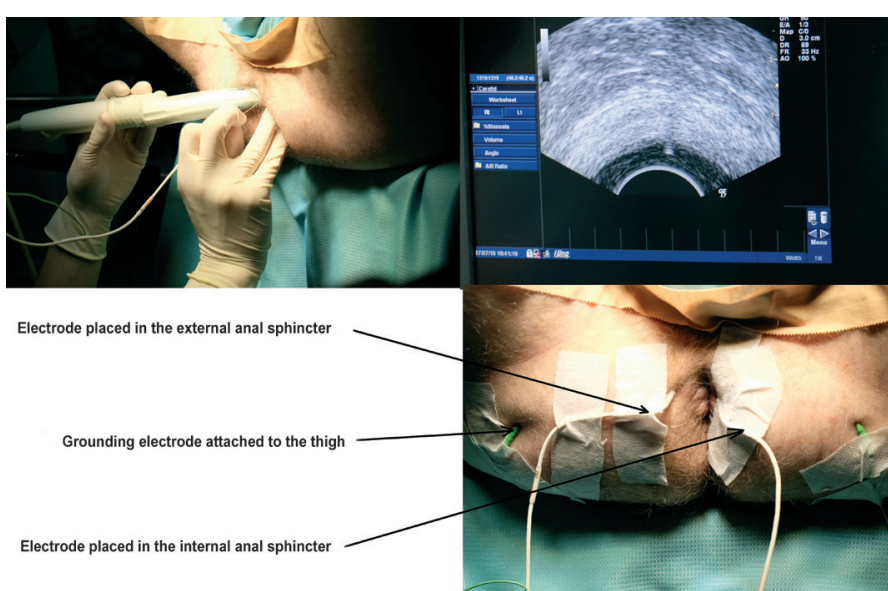

Fig. 1. Ultrasound-guided electrode placement in the sphincter complex

neurosurgery (5-9). The first attempts to identify the nerves of the pelvis were carried out with the CaverMap technique; however, it was characterized by poor repeatability and could be used only in men $(10,11)$. Employment of new precise techniques of nerve stimulation as well as combined recordings from the bladder and rectal sphincters give hope that neuromonitoring can be an effective tool for preservation of nerve function after surgery.

\section{AIM}

The aim of the study is to present, for the first time in Poland, our initial experience with modified intraoperative neuromonitoring 
A

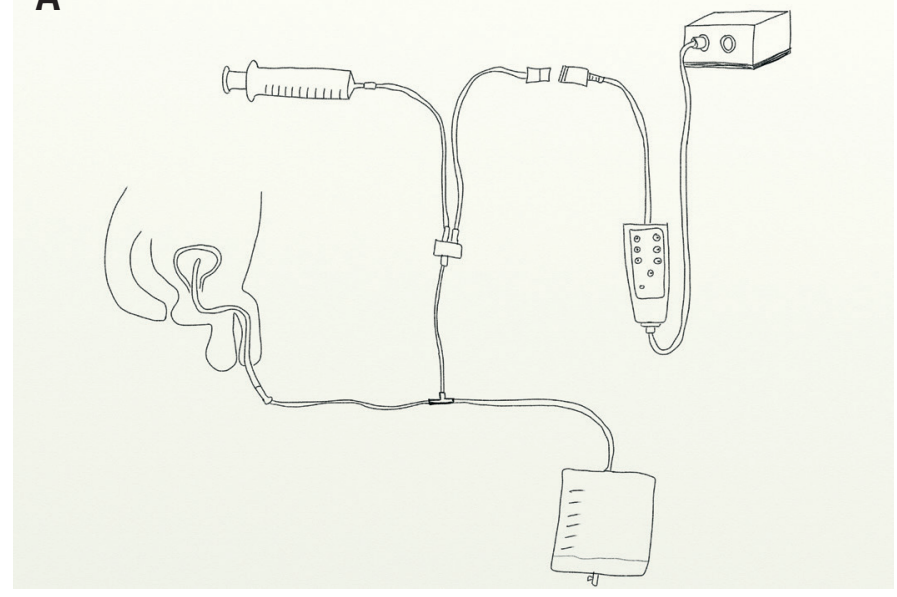

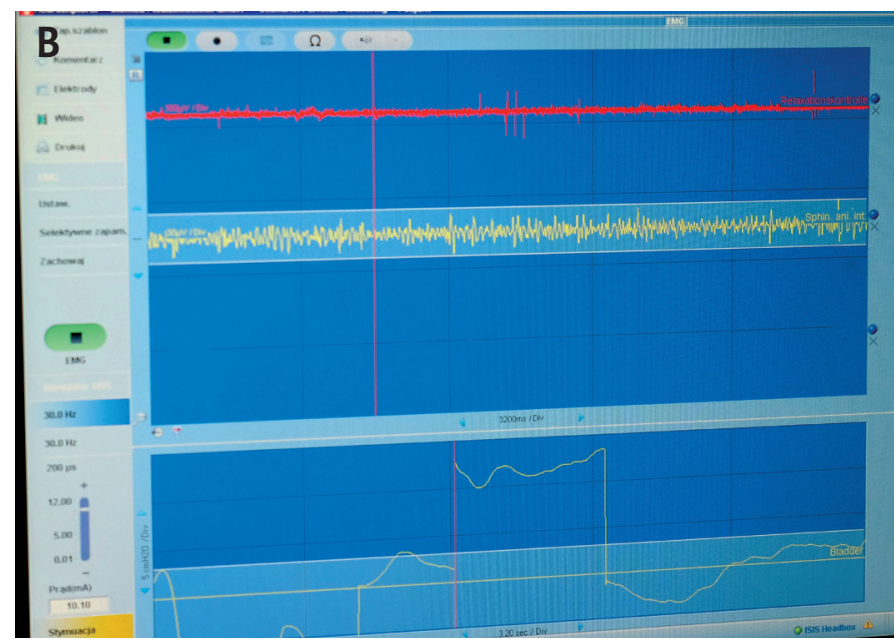

Fig. 2. Monitoring of bladder function. System of bladder pressure control (a) with intraoperative recordings (b)

of pelvic nerves during total mesorectal excision

\section{MATERIALS AND METHODS}

All procedures were performed in one center by the same operator who had substantial experience with regard to colon and rectal surgery. Members of the operating team, anesthesiologists, and auxiliary personnel were all trained with respect to preparation of equipment and patient preparation. The equipment was used by trained technicians. Four patients with rectal cancer (two men and two women, aged $42-53$ years) were enrolled. Anterior rectal resection was performed in 2 patients, low anterior rectal resection was performed in 1 patient, and proctocolectomy was performed in 1 patient. Patient characteristics and surgery duration times are presented in Table 1. In all patients, functional assessment of the anorectal areas was carried out before surgery. In contrast to the protocol developed by Kneist, preoperative superficial three-ring electromyography was performed with a 48-channel rectal probe (OT Bioelettronica, Torino), according to the method described by Merletti et al. (12). Patients were placed in the modified LoydDavies position, and needle EMG electrodes (15 $\mathrm{mm}$ in lengths, $0.5 \mathrm{~mm}$ in diameter) were placed under rectal ultrasound guidance in the external rectal sphincter at 4 and 6 o'clock (Fig. 1). Grounding electrodes were attached to the right thing. These electrodes were used for recording action potentials of the rectal sphincter complex. Moreover, we inserted a standard, 18FR Foley's urinary catheter to which a T-tube was connected to allow urine outflow and measurement of pressure changes in the bladder induced by detrusor contractions during stimulation. The diagram of the applied equipment is presented in Figure 2a.

Nerve stimulation was carried out with monophasic current (intensity, 3mA; frequency $30 \mathrm{~Hz}$, stimulus duration, $200 \mu \mathrm{s}$ ). For surgical procedures with the use of pelvic neuromonitoring, anesthesia protocols are changes, i.e., total intravenous anesthesia and non-depolarizing muscle relaxants are preferred.

\section{RESULTS}

During surgery, vital signs such as blood pressure, pulse, respiratory rate, and oxygen saturation remained normal in all patients. We did not observe intraoperative adverse effects. Setting up neuromonitoring prolonged surgery time by 30 to 40 minutes, or even by 60 to 80 minutes in the case of first two patients. Neuromonitoring itself took additional 20 to 30 minutes. This significantly prolonged surgery time, but one should take into account that we performed these procedures without prior experience. In all patients, stimulation of inferior hypogastric plexuses at the normal anatomic position induced symmetrical signals form the bladder and internal rectal sphincter (Fig. 3). In one patient with a tumor that extended beyond the lateral recital wall, dissection of the lateral part of the mesorectum on the left side caused loss of signals from the internal sphincter and the bladder on stimulating the left branches of the inferior hypogastric plexus, while the responses on stimulating the right branches of the same plexus remained normal. In that patient, we observed symptoms of neurogenic bladder after surgery. In the same patient, EMG signal on the left side was weaker, albeit present, on preoperative functional assessment (sphincter EMG, anorectal manometry). At the 3-month follow-up, the EMG signal of the left external rectal sphincter was reduced in comparison to the preoperative assessment, and we observed dysfunction of the reflex function of the internal rectal sphincter and dysfunction of micturition.

\section{DISCUSSION}

In 2004, prof. Kneist from Heidelberg published the first human study that used intraoperative monitoring of the nerves in the lesser pelvis. He performed TME in 17 patients with rectal cancer, and in two of those patients, Kneist did not record any intraoperative signals, which was accompanied by sustained disorders of micturition (13). According to the Heidelberg team, sensitivity, specificity, and positive and negative predictive values of the combined use of neuromonitoring and EMG for preservation of internal anal sphincter (IAS) innervation during rectal surgery are 100\%, 96\%, $67 \%$, and $100 \%$, respectively. Notably, neuromonitoring of pelvic nervous plexuses is different than neuromonitoring used in other fields of surgery, because pelvic nerves have different characteristics. In head and neck surgery, neuromonitoring is based on the stimulation of somatic nerves which induces immediate effector muscle response. That response is recorded by an electrode placed in the muscle, and it is displayed on a monitor and simultaneously recoded into an auditory signal. In the case of the pelvic nerves,, that are composed primarily of autonomic fibers, effector response 
is less potent and often prolonged in time. For that reason, pelvic nerve neuromonitoring resembles more neuromodulation than neurostimulation (13-16).

No intraoperative or postoperative complications of pelvic nerve monitoring have been noted. Although pelvic intraoperative neuromonitoring ( $\mathrm{pION}$ ) extended surgery time in our patients, one should remember of the learning curve. Because pION is only minimally invasive, no sustained complication or electrode-related adverse effects have been noted. The use of a superficial multichannel EMG probe before surgery instead of needle electrodes reduces patient discomfort.

Neuromonitoring is a method that was introduced over 100 years ago, but is has recently gained interest. Initially, it was used for the assessment of the somatic nervous system. Although we used neuromonitoring in patients with cancer, it can also be used in patients with other conditions, e.g. for rectopexy (Ripstein, Frykman-Golderg), or for pelvic surgical procedures that are performed in gynecology and urology. Neuromonitoring can be used for open as well as for laparoscopic surgery, and it allows to use a wide range of surgical equipment. German researchers have studied how the technique of operative dissection influences the quality of EMG signal from the IAS, and they have not found any significant differences between dissection performed with the use of scissors, monopolar electrocoagulation, and the ultrasound-based Ultracision system. Thus, the operator can freely choose surgery techniques.

Close cooperation between the surgeon, clinical neurophysiologist, and anesthesiologist is essential for a successful use of intraoperative neuromonitoring. Without proper anesthesia, the obtained signal can be unreliable.

Based on the available literature, lack of electromyographic signal does not necessarily indicate sphincter denervation. Schneider et al. have determined that the quality of electromyographic signal depends on several factors, such as electrode type, electrode placement, stimulation site, stimulation intensity, and stimulation time. The loss of electromyographic signal, that we observed unilaterally in one of our patients, could have been caused by electrode dislocation. However, sphincter dysfunction and dysfunction of micturition that were seen at follow-up confirmed intraoperative damage. Thus, there was a correlation between intraoperative damage and postoperative functional deficit. We plan a larger prospective trial in order to confirm this observation. How does intraoperative neuromonitoring influence postoperative sexual function? Knesit et al., in a study with 17 patients who underwent laparoscopy, showed that lack of intraoperative signal from the inferior hypogastric plexus was strongly associated with sexual dysfunction after surgery.

\section{REFERENCES}

1. Heald R.J., Husband E.M., Ryall R.D.: The mesorectum in rectal cancer surgery - the clue to pelvic recurrence? Br. J. Surg. 1982 Oct.; 69 (10): 613-616.

2. Heald R.J., Moran B.J., Ryall R.D.: Rectal cancer: the Basingstoke experience of total mesorectal excision, 1978-1997. Arch. Surg. 1998 Aug.; 133 (8): 894-849.

3. Bryant C.L., Lunniss P.J., Knowles C.H. et al.: Anterior resection syndrome. Lancet Oncol. 2012; 13 (9): 403-408.

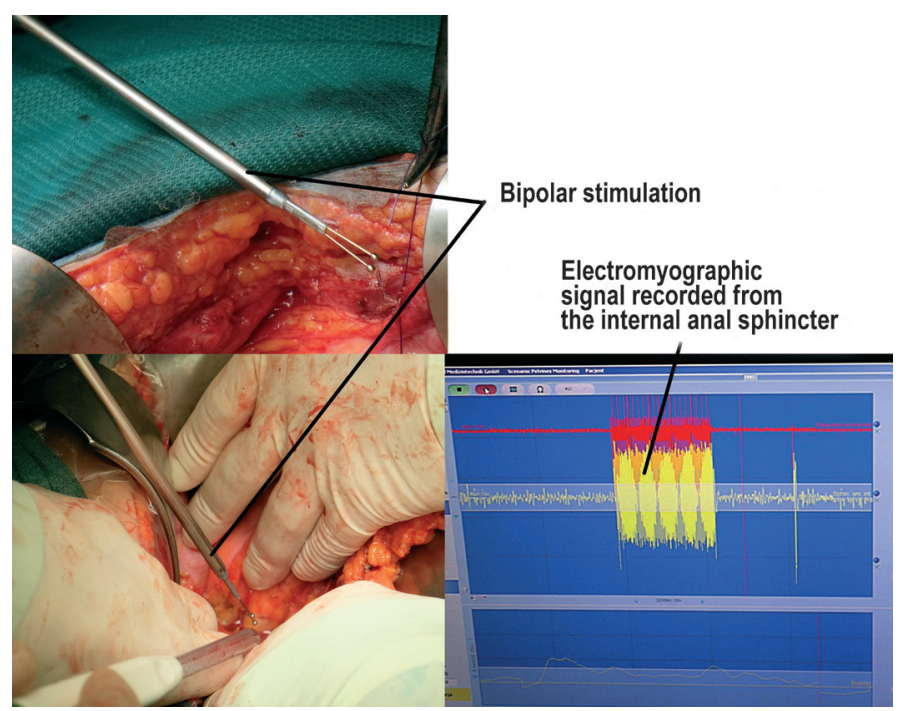

Fig. 3. Surgery in the phase of neuromonitoring.

The issue of prolonged surgery time, which is caused by the time needed to precisely place electrodes in the sphincter complex and to carry out stimulation in multiple planes and levels, is currently debated. As in the case of all new techniques, the learning curve plays an important role in this respect.

In conclusion, for the first time in Poland, we successfully employed intraoperative neuromonitoring during rectal surgery. Monitoring of bladder pressure and electromyographic signals from rectal sphincters enables visualization and preservation of autonomic nervous system structures, both sympathetic and parasympathetic. Intraoperative signals seem to be correlated with clinical presentation and functional examinations after surgery.

In order to objectify our results, it is necessary to perform multi-center studies in a larger sample. It is also necessary to perform functional examinations before and after surgery. It is especially important to compare sphincter electromyographic results obtained before, during, and after surgery in order to objectively determine the outcomes of neuromonitoring. Therefore, there is a need to create a standardized protocol for a multi-center trial in order to evaluate the procedure. Although neuromonitoring can initially prolong surgery time, mainly due to the time needed for ultrasound-guided electrode placement, this effect should decrease with progression of the learning curve.

\section{O FINANSOWANIU BADAŃ:}

Praca była współfinansowana z programu „Bony na innowacje” w ramach Regionalnego Programu Województwa Małopolskiego (nr K/KDU/000352) 
7. Guo L., Jasiukaitis P., Pitts L.H. et al.: Optimal placement of recording electrodes for quantifying facial nerve compound muscle action potential. Otol. Neurotol. 2008 Aug.; 29 (5): 710-713

8. Silverstein H., Rosenberg S.: Intraoperative facial nerve monitoring. Otolaryngol. Clin. North. Am. 1991 Jun.; 24 (3): 709-725.

9. Dralle H., Lorenz K.: Intraoperative neuromonitoring of thyroid gland operations: Surgical standards and aspects of expert assessment. Chirurg. 2010 Jul.; 81 (7) $612-619$

10. da Silva G.M., Zmora O., Börjesson L. et al.: The efficacy of a nerve stimulator (Cavermap) to enhance autonomic nerve identification and confirm nerve preservation during total mesorectal excision. Dis. Colon Rectum. 2005 Dec.; 48 (12): 2354-2361.

11. Kania D., Walega P.: Intraoperative mapping of pelvic autonomic nervous sys tem -a novel technique enabling preserwation of integrity of pelvic autonomic nervous system during rectal resection. Proktologia. 2008. 8: 193-206. Merletti R., Bottin A., Cescon C., Farina D., Gazzoni M., Martina S., Mesin L., Pozzo M., Rainoldi A., Enck P.: Multichannel surface EMG for the non-invasi- ve assessment of the anal sphincter muscle. Digestion. 2004; 69 (2): 112-122. Epub 2004 Apr. 14. PubMed PMID: 15087578

12. Kneist W., Heintz A., Junginger T.: Intraoperative identification and neurophysiologic parameters to verify pelvic autonomic nerve function during total mesorectal excision for rectal cancer. J. Am. Coll. Surg. 2004 Jan.; 198 (1): 59-66.

13. Kneist W., Junginger T.: Intraoperative electrostimulation objectifies the assessment of functional nerve preservation after mesorectal excision. Int. J. Colorectal. Dis. 2007 Jun.; 22 (6): 675-682.

14. Kneist W., Junginger T.: Male urogenital function after confirmed nerve-sparing total mesorectal excision with dissection in front of Denonvilliers' fascia World J. Surg. 2007 Jun.; 31 (6): 1321-1328.

15. Kneist W., Kauff D.W., Juhre V. et al.: Is intraoperative neuromonitoring associated with better functional outcome in patients undergoing open TME? Results of a case-control study. Eur. J. Surg. Oncol. 2013 Sep.; 39 (9): 994-999.

Word count: $1900 \quad$ Page count: $4 \quad$ Tables: $-\quad$ Figures: $4 \quad$ References: 15

DOI: $\quad 10.5604 / 01.3001 .0009 .9161$

Table of content: http://ppch.pl/resources/html/articlesList?issueld=9556

Copyright: Copyright @ 2017 Fundacja Polski Przegląd Chirurgiczny. Published by Index Copernicus Sp. z o. o. All rights reserved

Competing interests: The authors declare that they have no competing interests.

2 The content of the journal "Polish Journal of Surgery" is circulated on the basis

- of the Open Access which means free and limitless access to scientific data.

\section{(c) (1) () (2)} BY NC SA

Corresponding author:

Cite this article as:

This material is available under the Creative Commons - Attribution 4.0 GB. The full terms of this license are available on: http://creativecommons.org/licenses/by-nc-sa/4.0/legalcode

Piotr Wałęga Tel.: +48 126331995 E-mail: pwalega@mp.pl

Wałęga P., Romaniszyn M., Wałęga M, Świrta S., Nowak W.; Intraoperative neuromonitoring of hypogastric plexus branches during surgery for rectal cancer - preliminary report ; Pol Przegl Chir 2017: 89 (2): 69-72 